\title{
Simulation of bootstrap current in 2D and 3D ideal magnetic fields in Tokamaks
}

\author{
M. Raghunathan, ${ }^{1}$ J. P. Graves, ${ }^{1}$ W. A. Cooper, ${ }^{1}$ M. Pedro, ${ }^{1}$ and O. Sauter ${ }^{1}$ \\ École Polytechnique Fédérale de Lausanne (EPFL), Swiss Plasma Center (SPC), CH-1015 Lausanne, \\ Switzerland.
}

(Dated: 15 March 2016)

\begin{abstract}
We aim to simulate the bootstrap current for a MAST-like spherical tokamak using two approaches for magnetic equilibria including externally caused 3D effects such as Resonant Magnetic Perturbations (RMPs), the effect of toroidal ripple, and intrinsic 3D effects such as non-resonant internal kink modes. The first approach relies on known neoclassical coefficients in ideal MHD equilibria, using the Sauter[O. Sauter et al, Phys. Plasmas 6, 2834, (1999)] expression valid for all collisionalities in axisymmetry, and the second approach being the quasi-analytic Shaing-Callen[K. C. Shaing and J. D. Callen, Phys. Fluids 26, 3315, (1983)] model in the collisionless regime for 3D. Using the ideal free-boundary magnetohydrodynamic code VMEC, we compute the flux-surface averaged bootstrap current density, with the Sauter and Shaing-Callen expressions for 2D and 3D ideal MHD equilibria including an edge pressure barrier with the application of resonant magnetic perturbations, and equilibria possessing a saturated non- resonant $1 / 1$ internal kink mode with a weak internal pressure barrier. We compare the applicability of the self-consistent iterative model on the 3D applications and discuss the limitations and advantages of each bootstrap current model for each type of equilibrium.
\end{abstract}

\section{INTRODUCTION}

The bootstrap current plays an important role in the steady-state function of future fusion devices, especially tokamaks such as ITER, as it reduces the dependence on external current drive, leading to savings in the input energy. Thus, it is of great interest to study the bootstrap current in existing fusion devices, under operational conditions similar to ITER.

For tokamaks with steep edge pedestals, similar to those found in H-mode operations, the bootstrap current near the edge acquires a large value, significantly reducing the dependence on current drive. In TCV, operation of the tokamak with $100 \%$ bootstrap current has been demonstrated ${ }^{1}$, leading to hopes that future tokamaks can achieve high bootstrap-current fractions. At the same time, plasma behaviour at and near the edge is crucial for the operation of a tokamak. The toroidal field ripple caused by the discretization of the toroidal magnetic field is a $3 \mathrm{D}$ effect that can play an important role in confinement of particles near the edge. Another example of such 3D effect on the edge is the Edge Localized Mode (ELM), which in short bursts, causes large degradation to the confinement of the plasma. Recently, the effort towards mitigating ELMs has concentrated on using Resonant Magnetic Perturbations (RMPs), to mitigate and control ELMs.However, large density pumpouts associated with ELM mitigation can cause a change in the edge bootstrap current. In addition to the $3 \mathrm{D}$ effects caused by externally imposed magnetic fields, the saturated 1/1 internal kink mode, also known in experimental plasma physics as Long Lived Modes (LLMs), is an intrinsic effect in toroidally confined hybrid-type plasmas. The pressure barrier around the helical-core region can contribute significantly to the bootstrap current, and therefore, it is important to see to what extent the helical core affects the bootstrap current ordinarily associated with core localised pressure gradients.

In this paper, we attempt to undertake such selfconsistent calculations of the bootstrap current in the $3 \mathrm{D}$ applications mentioned above. In particular, we use a self-consistent iterative scheme for the bootstrap current and employ two contrasting models to compute the bootstrap current. The iterative scheme yields the bootstrap current self-consistently starting from an ideal (2D and 3D) MHD equilibrium obtained from the VMEC $\operatorname{code} e^{2}$. The two distinctive models of bootstrap current we use are as follows: First, the Sauter model ${ }^{3}$ and second the Shaing-Callen model ${ }^{4}$. Both bootstrap current calculation models are applied to both 2D and 3D equilibria, even though the Sauter model was originally derived for axisymmetric equilibrium calculations, and the ShaingCallen model was conceived for $3 \mathrm{D}$ equilibria. In the current work, we compare the performance of the two models for a variety of cases ranging from axisymmetric equilibria, to $3 \mathrm{D}$ equilibria incorporating the ideal response of RMPs to 3D equilibria with a helical core. This resultant bootstrap current is incorporated into the original toroidal current density profile, and iterated through VMEC again to generate a new equilibrium. The scheme is iterated until the bootstrap current profiles are sufficiently converged, resulting in a self-consistent magnetic equilibrium and its resultant bootstrap current profile. There have been apparently similar attempts to simulate bootstrap current using an iterative scheme on tokamaks and stellarators $5 \mid 6$. However, each work has usually limited itself to using either an axisymmetric model or solely using a 3D model. The current work will compare and contrast the two bootstrap current models on 2D and 3D equilibria, obtaining novel, realistic bootstrap current calculations in tokamaks with field ripple, RMPs and helical cores. 
The paper is organised as follows: In the first section, we describe the self-consistent approach to obtaining the bootstrap current from an ideal MHD equilibrium. In the second section, we benchmark the performance of the scheme for an axisymmetric equilibrium. In section 3 , we consider $3 \mathrm{D}$ equilibria with a steep edge pressure pedestal, and explore the effect of toroidal field ripple and the effect of RMPs on the bootstrap current. In section 4 , we study one case of helical-core $3 \mathrm{D}$ equilibrium and its associated bootstrap current density profiles. Brief concluding remarks are provided in section 5 .

\section{APPROACH TO THE SIMULATION AND BOOTSTRAP CURRENT MODELS}

Our aim to calculate the bootstrap-current and a magnetic equilibrium consistently requires that if the initial current profile used for the magnetic equilibrium calculation included the bootstrap current, the equilibrium thus generated would extract the same bootstrap current density profile as the one we began with. In order to establish this iteratively, we need a magnetic equilibrium and an interface for calculating the bootstrap current. The equilibrium for the iterative process is provided by the Variational Moments Equilibrium Code (VMEC) ${ }^{2}$. VMEC is a versatile ideal free-boundary MHD equilibrium code which generates equilibrium by minimizing the variations in the equilibrium energy functional. Using the free-boundary version of $\mathrm{VME} \mathrm{C}^{8}$, we generate equilibria for the desired current and pressure profiles. We also vary the equilibrium by specifying an initial guess of a skewed magnetic axis of $1 / 1$ helicity with axisymmetric boundary conditions that leads to the formation of a helical core, thereby allowing the representation of a $1 / 1$ internal kink mode ${ }^{12}$. One can include the effect of external fields in VMEC by prescribing the coil positions and coil currents. A package within the VMEC-Suite, MAKEGRID allows us to calculate the magnetic field generated by the specified coils. Including this external magnetic field in the equilibrium calculations allows us to generate equilibria with the ideal response of the equilibrium to the external fields. One element of this study is to analyse the the variation in the equilibrium and the bootstrap currents due to the varying number of toroidal field coils (TF-coils), and due to the Resonant Magnetic Perturbation (RMP) coils. In addition to the externally produced
3D effects, VMEC can also generate a realistic representation of a saturated $1 / 1$ internal kink mode ${ }^{13}$. Of special interest will be to examine and contrast the $3 \mathrm{D}$ helical core state and the axisymmetric sister-state and thus isolate the $3 \mathrm{D}$ effect of the bootstrap current.

For calculating the bootstrap current, we consider two models. First, the Sauter model ${ }^{3}$, which is an axisymmetric 2D model, and the second is the Shaing-Callen mode ${ }^{4 / 7}$ which is quasi-analytic and valid for $3 \mathrm{D}$ equilibria. The calculation using these models is performed in a separate module. The advantage of this separate module is that it can take into account the specific profiles for each numerical experiment, thus delivering a tailored bootstrap current profile for each particular simulation.

We now proceed to describe the Sauter bootstrap current model in subsection IIA, the Shaing-Callen bootstrap current model in subsection IIB explaining the resonance effects and resonance detuning (in IIC), and the iterative scheme used for the self-consistent bootstrap current calculation in subsection IID.

\section{A. The Sauter bootstrap current density model}

The expression given by Sauter et al, in which the parallel bootstrap current density $\left\langle\mathbf{J}_{\mathbf{b s}} \cdot \mathbf{B}\right\rangle$, is given by

$$
\left\langle\mathbf{J}_{\mathbf{b s}} \cdot \mathbf{B}\right\rangle=-I(\psi) p_{e}\left[L_{31} \frac{p}{p_{e}} \frac{\partial \ln p}{\partial \psi}+L_{32} \frac{\partial \ln T_{e}}{\partial \psi}+L_{34} \alpha \frac{\partial \ln T_{i}}{\partial \psi}\right]
$$

where $\mu_{0} I(\psi)=-B_{v}$ in VMEC coordinates (or equivalently $\mu_{0} I(\psi)=R B_{\phi}$ assuming an axisymmetric magnetic field). Throughout the current work, we work in S. I. units for the sake of consistency through all our simulations. Following usual conventions, $\psi$ is the fluxsurface label, $p$ is the pressure and $T_{j}$ is the temperature of the species $j$ in the plasma. The dimensionless factor $\alpha$ is a coefficient for correctly accounting for the contribution of each species towards the bootstrap current in the collisionless limit.

The coefficients $L_{31}, L_{32}, L_{34}$ and $\alpha$ are described in Ref. 3 where these are determined as fits of functions of the trapped fraction of particles and collisionalities. After the fit to the previously computed results in Ref. 3, these have the following expressions in terms of the trapped particle fraction $f_{t}$ in the $1 / \nu$ collisionless regime:

$$
\begin{aligned}
L_{31}= & \left(1+\frac{1.4}{Z+1}\right) f_{t}-\frac{1.9}{Z+1} f_{t}^{2}+\frac{0.3}{Z+1} f_{t}^{3}+\frac{0.2}{Z+1} f_{t}^{4} \\
L_{32}= & {\left[\frac{0.05+0.62 Z}{Z(1+0.44 Z)}\left(f_{t}-f_{t}^{4}\right)+\frac{1}{1+0.22 Z}\left(f_{t}^{2}-f_{t}^{4}-1.2\left(f_{t}^{3}-f_{t}^{4}\right)\right)+\frac{1.2}{1+0.5 Z} f_{t}^{4}\right] } \\
& +\left[-\frac{0.56+1.93 Z}{Z(1+0.44 Z)}\left(f_{t}-f_{t}^{4}\right)+\frac{4.95}{1+0.44 Z}\left(f_{t}^{2}-f_{t}^{4}-0.55\left(f_{t}^{3}-f_{t}^{4}\right)\right)-\frac{1.2}{1+0.5 Z} f_{t}^{4}\right]
\end{aligned}
$$


and

$$
\begin{aligned}
L_{34} & \approx L_{31} \\
\alpha\left(\nu_{*}=0\right) & =\alpha_{0}=\frac{1.17\left(1-f_{t}\right)}{1-0.22 f_{t}-0.19 f_{t}^{2}}
\end{aligned}
$$

where in $L_{32}$, the two terms in their respective square brackets represent the electron and ion contributions to $L_{32}$ respectively. $Z$ refers to the effective screened charge of the ions. In accordance to previous work, we set $Z=$ 1 throughout our simulations neglecting any screening effect. The trapped fraction of particles $f_{t}$ is computed as

$$
f_{t}=1-\frac{3}{4} \frac{\left\langle B^{2}\right\rangle}{B_{\max }^{2}} \int_{0}^{1} \frac{\lambda}{\left\langle g_{1}\right\rangle} d \lambda,
$$

where $g_{1}$ is given by

$$
g_{1}=\sqrt{1-\lambda \frac{B}{B_{\max }}},
$$

where the angle brackets $\langle x\rangle$ represent the quantity $x$ averaged over a flux-surface. Heretofore, we refer to Eqs. (1)-(5) as the Sauter formula.

\section{B. The Shaing-Callen bootstrap current density model}

The Shaing-Callen formulation is given by

$$
\left\langle\mathbf{J}_{\mathbf{b s}} \cdot \mathbf{B}\right\rangle=-G_{b}(\psi)\left[L_{31} \frac{\partial p}{\partial \psi}+L_{32}^{e} n_{e} \frac{\partial T_{e}}{\partial \psi}+L_{32}^{i} n_{i} \frac{\partial T_{i}}{\partial \psi}\right]
$$

where $G_{b} \equiv G_{b}(\psi)$ is a geometrical factor, calculated through averaging over the entire $3 \mathrm{D}$ field. The coefficients $L_{31}$ and $L_{32}^{i, e}$ are analytically determined in terms of the neoclassical viscosity coefficients and the trapped particle fractions. The expressions for the $L$-coefficients can be found in Ref. 17. In this sense, the Shaing-Callen formulation can be considered to be a quasi-analytic approach to determining the bootstrap current.

The geometrical factor $G_{b}(\psi)$ is computed in the $1 / \nu$ (collisionless) regime as

$$
G_{b}(\psi)=\frac{1}{f_{t}}\left\{\left\langle g_{2}\right\rangle-\frac{3}{4} \frac{\left\langle B^{2}\right\rangle}{B_{\max }^{2}} \int_{0}^{1} \frac{\left\langle g_{4}\right\rangle}{\left\langle g_{1}\right\rangle} \lambda d \lambda\right\}
$$

where again,

$$
\begin{aligned}
f_{t} & =1-\frac{3}{4} \frac{\left\langle B^{2}\right\rangle}{B_{\max }^{2}} \int_{0}^{1} \frac{\lambda}{\left\langle g_{1}\right\rangle} d \lambda, \\
g_{1} & =\sqrt{1-\lambda \frac{B}{B_{\max }}} .
\end{aligned}
$$

The quantities $g_{2}$ and $g_{4}$, in turn, must also satisfy the following expressions.

$$
\begin{aligned}
\text { B. } \nabla\left(\frac{g_{2}}{B^{2}}\right) & =\mathbf{B} \times \nabla \Phi \cdot \nabla\left(\frac{1}{B^{2}}\right) \\
\text { B. } \nabla\left(\frac{g_{4}}{g_{1}}\right) & =\mathbf{B} \times \nabla \Phi \cdot \nabla\left(\frac{1}{g_{1}}\right) \\
g_{2}\left(B_{\max }\right) & =0 \\
g_{4}\left(B_{\text {max }}\right) & =0
\end{aligned}
$$

where $\Phi$ is the toroidal flux, related to the poloidal flux $\psi$ through the safety factor $q=d \Phi / d \psi$ (thereby making $\nabla \psi$ and $\nabla \Phi$ canonical flux coordinates). We integrate these equations by transforming them into Fourier-space, where the gradients can be realized simply as coefficients multiplying the Fourier-transformed integrand.

\section{Numerical resonance mitigation}

The mitigation of numerical resonances at rational $q$ surfaces is of particular importance to the Shaing-Callen mode 19 . For solving the equations in the Shaing-Callen model, we use a Fourier-decomposition scheme to simplify the equations in Fourier space. However, the B. $\nabla$ operator is proportional to $\left(m \Psi^{\prime}-n \Phi^{\prime}\right)^{-1}$, which is singular at rational $q=m / n$ surfaces. This has been mitigated in previous work by the inclusion of a resonance detuning operator $\Delta_{m n}$, which numerically prevents the singularities from occurring. The detuning operator is defined as follows:

$$
\Delta_{m n}=\Delta\left[(m+1) \Psi^{\prime}-n \Phi^{\prime}\right]
$$

where $\Delta$ is a dimensionless factor determining the amplitude of the resonance detuning. The singularity itself is prevented by changing its form to

$$
\frac{1}{m \Psi^{\prime}-n \Phi^{\prime}} \rightarrow \frac{m \Psi^{\prime}-n \Phi^{\prime}}{\left(m \Psi^{\prime}-n \Phi^{\prime}\right)^{2}+\Delta_{m n}^{2}} .
$$

It is important to note that this scheme is purely a numerical correction on the resonant $q=m / n$ surfaces. In reality, these resonances represent parallel current sheets which would create islands and local pressure flattening (and thus reduction of the local bootstrap current density) in a resistive MHD model. However, this cannot be accounted for by an ideal MHD equilibrium code like VMEC, and hence is unphysical under the VMEC equilibrium model. As will be seen, the $1 / 1$ non-resonant internal kink mode is a particularly interesting application because the core 3D structure avoids resonance and hence, the need of the resonance detuning here.

In the bootstrap current density profile, the resonant contributions appear as sharp spikes at the values of $s$ corresponding to the resonant $q$-values. These spikes are very sensitive to the choice of the detuning factor $\Delta$. Choosing too large a value of $\Delta$ makes the current density profile globally distorted to a significant order, and the choice of too small $\Delta$ leads to the presence of large spikes at rational $q$ surfaces. We will explore this in more detail in the next section. 


\section{Computing the bootstrap current}

In order to calculate the flux-surface averaged toroidal bootstrap current density $\left\langle\mathbf{J}_{\mathbf{b s}} \cdot \nabla \phi\right\rangle(\psi)$, we must average over the toroidal and poloidal angles as follows

$$
\left\langle\mathbf{J}_{\mathbf{b s}} \cdot \nabla \phi\right\rangle(\psi)=\int_{0}^{2 \pi} \int_{0}^{2 \pi} \frac{\left\langle\mathbf{J}_{\mathbf{b s}} \cdot \mathbf{B}\right\rangle(\psi)}{B^{2}} \Phi^{\prime}(\psi) \mathrm{d} u \mathrm{~d} v
$$

where again the angled brackets $\langle x\rangle$ are used to represent the flux-surface average of the parameter $x$. In VMEC coordinates, this is computationally difficult to perform on account of coordinate system used. The toroidal current density $\left\langle\mathbf{J}_{\mathrm{bs}} \cdot \nabla \phi\right\rangle$ in VMEC coordinates is given by

$$
\left\langle\mathbf{J}_{\mathbf{b s}} \cdot \nabla \phi\right\rangle(\psi)=\left\langle\mathbf{J}_{\mathbf{b s}} \cdot \mathbf{B}\right\rangle(\psi) \int_{0}^{\pi} \int_{0}^{2 \pi} \frac{\mathrm{d} u \mathrm{~d} v \sqrt{g}\left(\Phi^{\prime}-\frac{\partial \lambda}{\partial u}\right) g^{v v}}{\left(\Psi^{\prime}+\frac{\partial \lambda}{\partial v}\right)^{2} g_{u u}+\left(\Phi^{\prime}-\frac{\partial \lambda}{\partial u}\right)^{2} g_{v v}+2\left(\Psi^{\prime}+\frac{\partial \lambda}{\partial v}\right)\left(\Phi^{\prime}-\frac{\partial \lambda}{\partial u}\right) g_{u v}} .
$$

This is computationally expensive to calculate for each flux-surface and over each iteration of the scheme, as the double integral would have to performed for every point of the defined grid in $u$ and $v$ with sufficient precision. Instead, we convert the obtained equilibrium to Boozer coordinates using TERPSICHORE ${ }^{15}$. On multiplying the numerator and the denominator by the Jacobian $\sqrt{g}$, we find that the averaging in Eq. (16) is just required over $\left\langle\mathbf{J}_{\mathbf{b s}} \cdot \mathbf{B}\right\rangle$ as follows

$$
\left\langle\mathbf{J}_{\mathbf{b s}} \cdot \nabla \phi\right\rangle(\psi)=\left\langle\mathbf{J}_{\mathbf{b s}} \cdot \mathbf{B}\right\rangle(\psi) \frac{\Phi^{\prime}(s) V^{\prime}(s)}{\Psi^{\prime}(s) J(s)-\Phi^{\prime}(s) I(s)} .
$$

It is immediately noticeable that the computation required to perform the integral has been reduced by a factor of $n_{u} \times n_{v}$, where $n_{u}$ and $n_{v}$ are the grid sizes chosen over the VMEC coordinates $u$ and $v$. Now one can integrate over the value of $\left\langle\mathbf{J}_{\mathbf{b s}} \cdot \nabla \phi\right\rangle$ to arrive at the value of bootstrap current profile $I_{B S}(\psi)$ as follows:

$$
I_{b s}(\psi)=\int_{0}^{\psi}\left\langle\mathbf{J}_{\mathbf{b s}} \cdot \nabla \phi\right\rangle(\psi) d \psi
$$

where $I_{b s}$ is the total bootstrap current obtained in amperes. In addition, the net toroidal current density has to be adjusted for the bootstrap current for the next iteration of the scheme in order to keep the total toroidal plasma current $I_{p}$ constant. If $\left\langle\mathbf{J}_{\mathbf{O h m}} \cdot \nabla \phi\right\rangle(\psi)$ is the purely Ohmic current density profile chosen over the first iteration of the equilibrium, the bootstrap current and the Ohmic current profiles are now modified so as to preserve the total toroidal current as follows:

$$
\langle\mathbf{J} . \nabla \phi\rangle(\psi)=\left(\frac{I_{p}-I_{B S}}{I_{\text {Ohm }}}\right)\left\langle\mathbf{J}_{\mathbf{O h m}} \cdot \nabla \phi\right\rangle(\psi)+\left\langle\mathbf{J}_{\mathbf{b s}} \cdot \nabla \phi\right\rangle(\psi)
$$

The coefficient of $\left\langle\mathbf{J}_{\mathbf{O h m}} \cdot \nabla \phi\right\rangle(\psi)$ is used to rescale the Ohmic current $I_{O h m}$ to match the desired value $I_{p}-I_{B S}$ from the values obtained at the previous iteration. This coefficient converges to 1 . At each iteration, the form of the Ohmic current is kept the same, but the overall current profile changes with respect to the form of the bootstrap current obtained in the last equilibrium. For the next iteration, VMEC takes the profile $2 \pi\langle\mathbf{J} . \nabla \phi\rangle(\psi)$ and creates a new equilibrium satisfying that profile.

In order for the iterative scheme to end, we stop it after an iteration where the bootstrap current converges to a sufficient precision. To that effect, we define the tolerance ' $t o l$ ' as the relative difference between the current density profiles between successive iterations. Therefore, we have for the $n^{\text {th }}$ iteration

$$
t o l=\frac{I_{B S}^{(n)}-I_{B S}^{(n-1)}}{I_{B S}^{(n-1)}}
$$

where the superscript $n$ represents the total bootstrap current at the $n^{\text {th }}$ iteration. We declare the bootstrap current density as having being 'saturated' when the specified tolerance is reached.

\section{BOOTSTRAP CURRENT PROFILES FOR 2D AND 3D EQUILIBRIA WITH A STEEP EDGE PRESSURE PEDESTAL}

We begin by generating equilibria with an input pressure profile. We choose the pressure profile in a manner so as to represent the steep edge pedestals observed in $\mathrm{H}$-modes in tokamaks (alternatively referred to as edge pressure 'barrier', as such steep profiles near the edge provide an edge transport barrier increasing the confinement of the plasma). The chosen pressure profile allows for an edge pressure pedestal beyond $s=0.8$ (where $s$ is the normalized toroidal flux given by $s=\Phi / \Phi_{\text {edge }}$ ), which can be seen in Fig. (1) (above). Further, the density profile is deliberately chosen to be a similar form as the pressure profile so as to obtain a smooth and relatively flat temperature profile near the edge. The temperature profiles is calculated as $T(s)=p(s) /(2 n(s))$, and as can be observed from Fig. (1) (below), is relatively flattened in the edge region beyond $s=0.8$. Additionally, we fix the density on the axis to $n_{0}=10^{20} \mathrm{~m}^{-3}$ and the temperature on the axis to be $T_{0}=0.6 \mathrm{keV}$. These values ensure faithful representation of the kind of equilibria seen in MAST, as can be together observed in Fig. (1). 

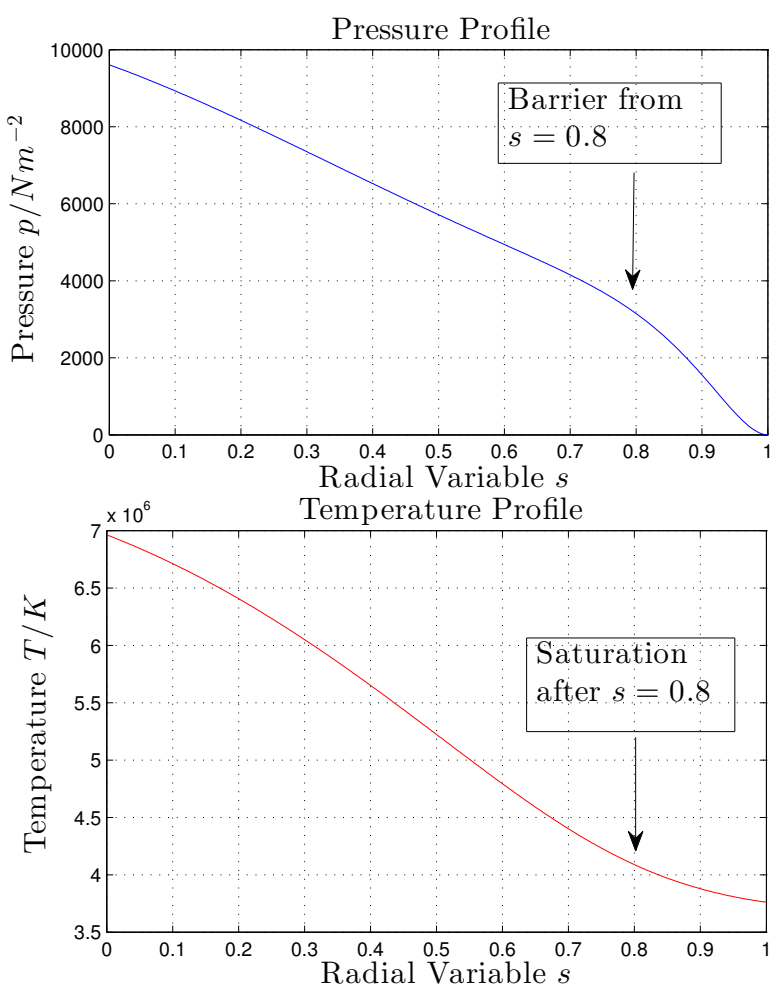

FIG. 1. The profiles are chosen so as to generate a VMEC equilibrium a steep edge pressure pedestal. (a) Notice the pressure barrier staring at $s=0.8$, and (b) The temperature profile flattens at $s>0.8$ so as to allow pedestals in pressure and density.

We begin the first iteration of the iterative scheme by setting solely the Ohmic part as the total toroidal current $I$. The initial Ohmic current profile is chosen as

$$
\left\langle\mathbf{J}_{\text {Ohm }} \cdot \nabla \phi\right\rangle(s)=I_{\text {total }} \frac{5}{12}\left(1-s-s^{2}+s^{3}\right) A m^{-2}
$$

and we fix the total toroidal plasma current to $I_{\text {total }}=$ $0.48 M A$ (where the $5 / 12$ is the normalization factor for the chosen $s$ polynomial). This prescription of pressure, density and temperature profiles is the initial condition for the calculation of the first equilibrium. The choice of our current density and pressure profiles determines the $q$-profile which plays an important role in the determination of the resonant rational surfaces which affects the bootstrap current densities calculated from 3D approaches. The associated $q$-profile can be seen in Fig. 2 With these profiles and axis parameters, we generate an equilibrium using VMEC. It is important to point out that the $(s, u, v)$ coordinates of VMEC make calculations easy wherever harmonic decomposition is necessary. However, for the calculation of the bootstrap current, these coordinates necessitate additional averaging loops because of the fact that these are not field-aligned coordinates. This is computationally expensive. Therefore, as alluded to in the previous section, we convert the equilibrium to Boozer coordinates through the use of the TERPSICHORE

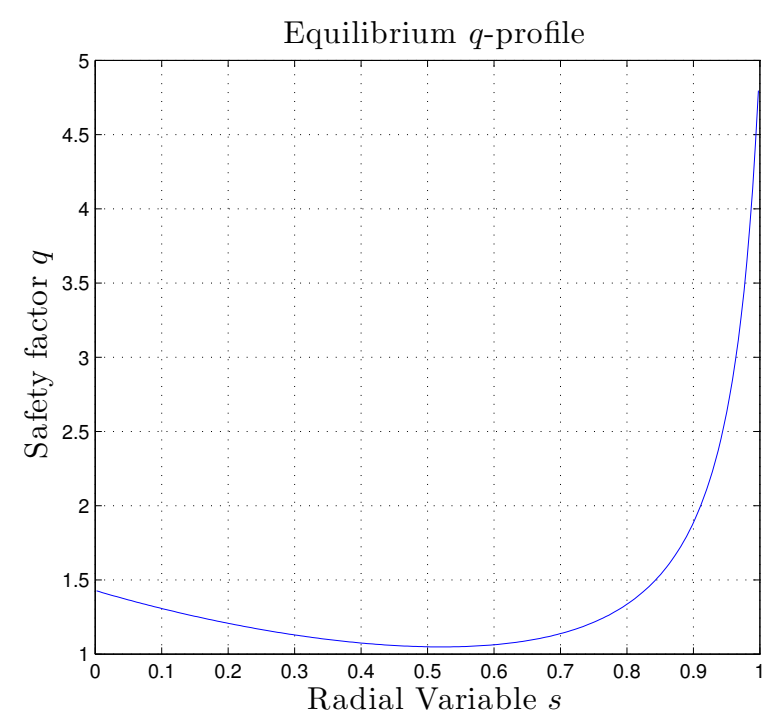

FIG. 2. The safety factor $q$-profile for the simulations for the chosen initial current and pressure profiles.

package 20 . The bootstrap current is then calculated through the use of the Sauter and Shaing-Callen formulae. The key idea, after this step is to scale and incorporate the bootstrap-current into the Ohmic current, thus prescribing the new current profile for the next iteration. This is subsequently iterated over with VMEC in order to generate a new equilibrium. The iterations are performed until convergence is reached to a required tolerance.

\section{A. Benchmark with an axisymmetric MAST equilibrium}

In order to benchmark the scheme, we first confine ourselves to axisymmetry. The VMEC free-boundary version is used to generate an axisymmetric equilibrium by allowing no toroidal modes except $n=0$. This also ensures that there are no resonant surfaces on which there can be singularities. Thus, we have the advantage of being able to examine the two bootstrap current models without the numerical effects of 3D magnetic equilibria. This lets us compare the forms and magnitudes of the bootstrap current generated by each model.

In Fig. 3 it is seen that even just after the first iteration, the Sauter and Shaing-Callen formulations prescribe bootstrap current density profiles which lie very close to each other, and follow the overall same shapes (which depend on the initial profiles we specified). In fact, the Shaing-Callen bootstrap current density is within $1 \%$ of the Sauter bootstrap current density. Therefore, we see that despite being prescribed by different schemes, one based on a fit and the other being quasi-analytic formulae, the profiles lie very close to each other.

Furthermore, it is of some interest to study the convergence of this scheme. In order to do so, as seen in Fig. (4), we iterate the scheme several times until con- 


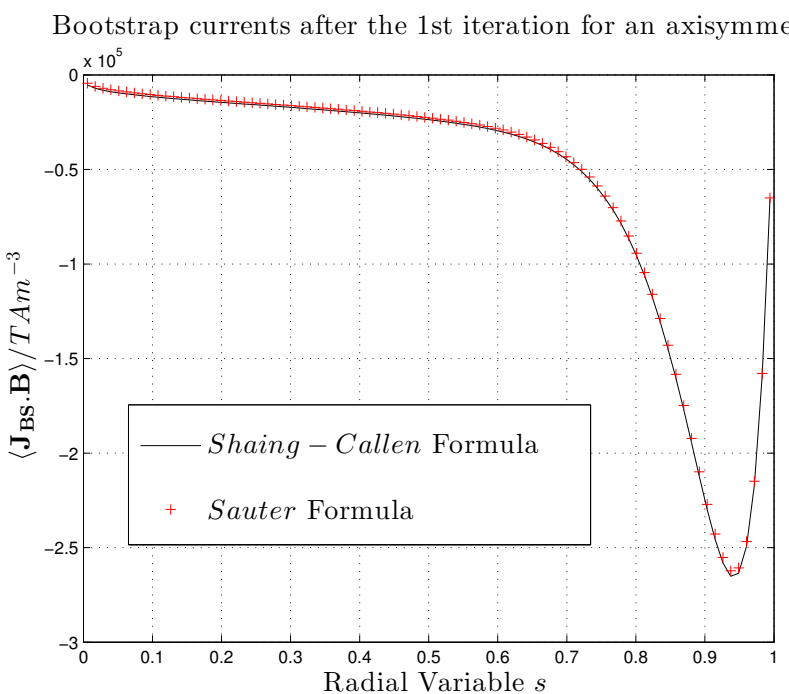

FIG. 3. Profiles of the parallel bootstrap current density $\left\langle\mathbf{J}_{\text {BS.B }}\right\rangle$ obtained according to the Sauter formula (the blue curve) and the Shaing-Callen formula (green '+' points).

vergence is reached to a required tolerance. In general, we notice that the Shaing-Callen scheme follows the Sauter scheme very closely. Thus, it is usually enough to seek convergence with respect to one of the prescriptions for the bootstrap current. On defining the tolerance as the relative difference between the current and the previous iteration, we seek a tolerance of $10^{-3}$. We observe that, with each iteration, the bootstrap current approaches saturation. Beyond the fourth iteration, the tolerance is achieved, and the current profile can be considered to be sufficiently converged.

Another point which is very useful to consider is that even at the end of the second iteration, the form for the bootstrap current lies very close to the final form of the bootstrap current density profile. For computation of the bootstrap current density for 3D equilibria, which are computationally expensive, one can use this fact as a simple test to obtain an idea of the form of the bootstrap current profile, before proceeding to iterate and obtaining the profile more precisely.

\section{B. Bootstrap current for a 3D equilibrium}

In order to look at 3D effects on the bootstrap current density and to compare the 3D models for the bootstrap current density, we generate MAST-like 3D equilibria using VMEC under free-boundary conditions, keeping the same pressure, temperature, Ohmic current and rotational-transform $\iota$ profiles. For our 3D case with possible resonant $q$ surfaces, we set the detuning factor $\Delta=10^{-4}$, the number of toroidal field coils (TF-coils) is chosen to be 12 consistently with the experiment, and the RMP coil current is set to $0 k A$ as a control. More details regarding the finite number of TF-coils and the

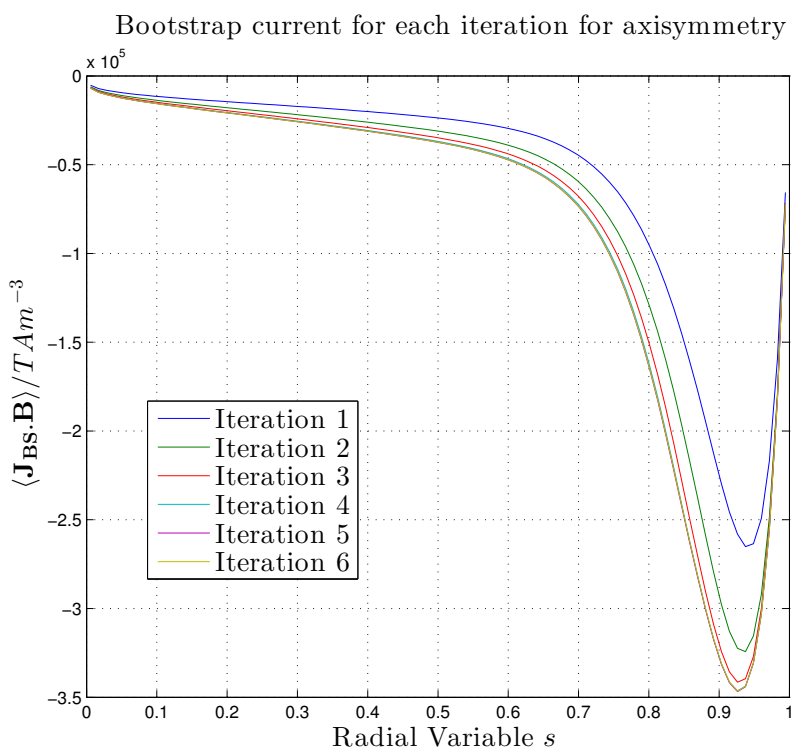

Total current for bootstrap iterations for axisymmetry

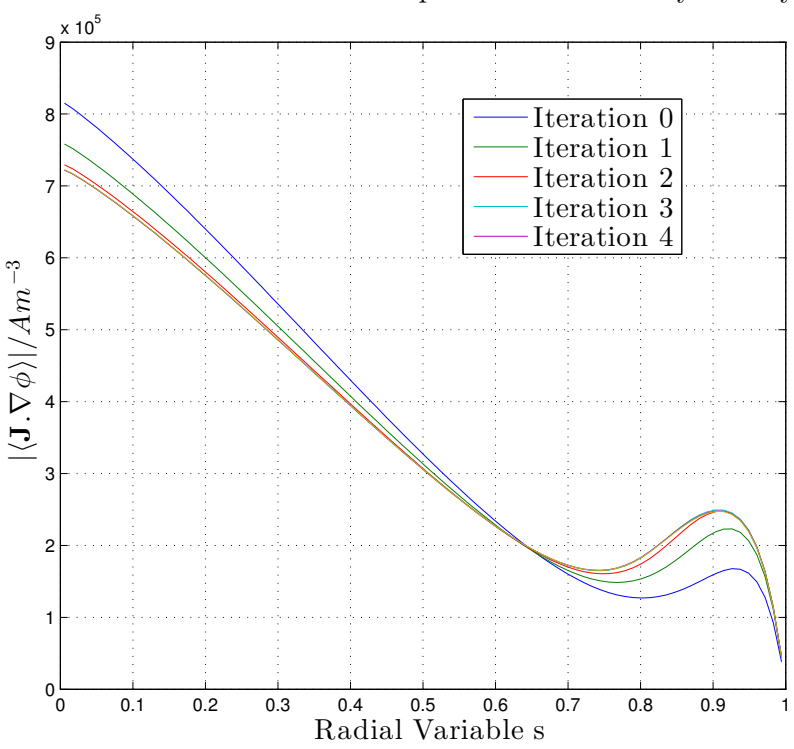

FIG. 4. Plots of the total current density profile and bootstrap current density profile assuming the Sauter formulation, showing variation at each iteration for an axisymmetric MAST equilibrium. (a) Plot of Sauter parallel bootstrap current density $\left\langle\mathbf{J}_{\mathbf{B S}}\right.$.B $\rangle$ for each iteration of the bootstrap procedure. (b) The total current profile $\langle\mathbf{J} . \nabla \Phi\rangle$ for each iteration of the bootstrap procedure. The profile for each $n^{t h}$ iteration is the input for the VMEC equilibrium calculation of the $(n+1)^{t h}$ iteration.

RMP coil current amplitude is provided below.

We can see in Fig. 5 that the Shaing-Callen derived bootstrap current now carries deviations from the axisymmetric Sauter model, as is expected from the 3D nature of the magnetic field. The spikes observed are caused by resonant rational $q$-surfaces, and in reality, represent 
Bootstrap currents after the 1st iteration for the $3 \mathrm{D}$ case

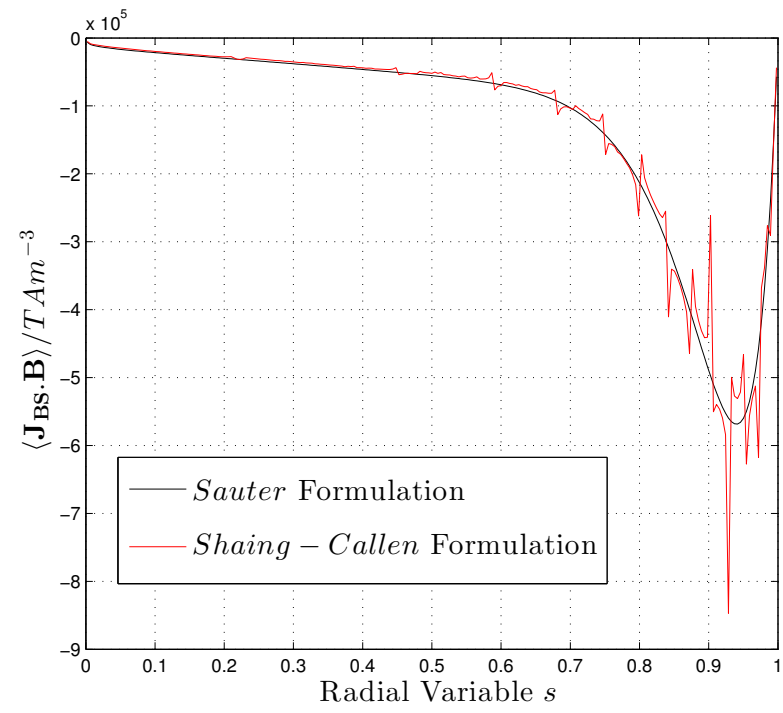

FIG. 5. Comparison of the Shaing-Callen formula and the Sauter formula for bootstrap current densities after the first iteration.

parallel sheet currents, as seen in the side-by-side comparison in Fig. (6). It can be immediately noticed that there are major resonances distorting the edge bootstrap current profile at and around the $q=2 / 1$ and $q=3 / 1$ surfaces. These resonances are not avoidable because of the choice of the mode numbers and the effectively attained $q$-profile. Additionally, we mention that the resonances have little effect on the convergence rate of the scheme. Furthermore, the choice of the grid for averaging over the pitch $\lambda$ in Eq. 9 plays little to no effect on the resonant spikes. We chose to implement several forms (linear, sinusoidal, hyperbolic) pitch grid between $s, \lambda$ so as to concentrate a high sampling density near the bounce point. We observe virtually no mitigation of the resonances.

However, we would still like to see whether these spikes can be removed by adequately adjusting the detuning factor $\Delta$. In Fig. (7), where the value of $\Delta$ is varied, we see that above $\Delta=10^{-4}$, the detuning causes the whole current density profile to change, distorting the current density profile itself. We observe, that the change in the profile becomes worse at $\Delta=10^{-2}$. And as we decrease below $\Delta=10^{-4}$, we observe that the profile remains the same. Thus, the optimal value of the detuning factor seems to be $10^{-4}$. However, we still see resonance causing spikes despite there being an optimal range for the resonance detuning 18 .

We now investigate the effect of toroidal field ripple. In what follows, the RMP currents are kept at $0 k A$ to obtain $3 \mathrm{D}$ effects solely from the toroidal field ripple created by the variation in the number of the toroidal field coils (TFcoils). In order to investigate whether resonance stemming from the toroidal field ripple can be suppressed,
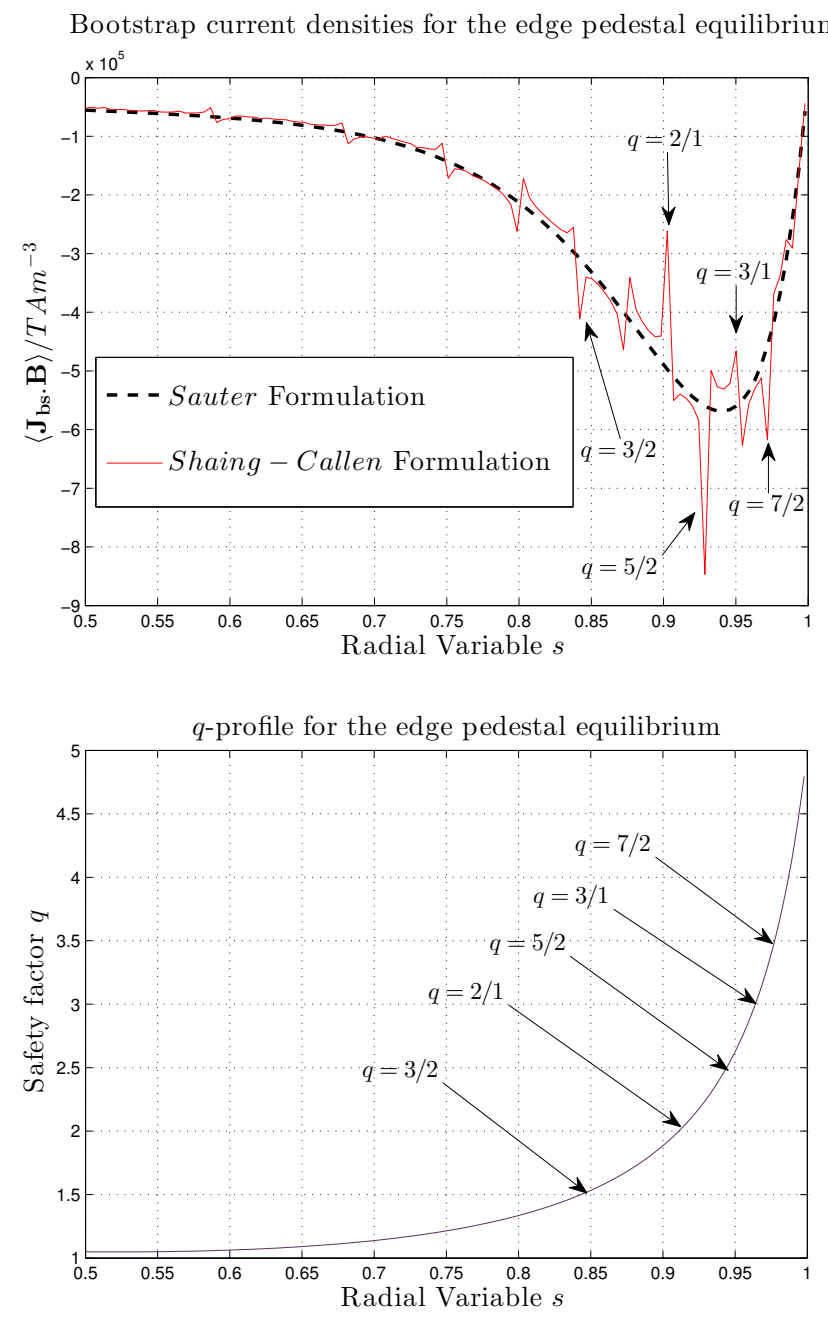

FIG. 6. A comparison of the Sauter and Shaing-Callen bootstrap current densities. It can be seen that the spikes on the edge bootstrap current correspond to rational values of $q$ on the profile.

we observe the change in bootstrap current density profile for increasing number of TF-coils with free-boundary calculations with VMEC. The normal number of TF-coils in MAST is 12. We investigate cases with 8 TF-coils (increased ripple), and with $12 \mathrm{TF}$-coils (ripple similar to observations in MAST), 24 and 32 TF-coils (decreased ripple).

The coil positions can be specified to VMEC using another package in the VMEC-Suite called MAKEGRID. MAKEGRID can define the magnetic-field strength of the TF-coils to any specified precision and to any specified number of modes. This is an important point to keep in mind, as increasing the number of coils would subsequently require an increase in the number of requested toroidal modes, in order to maintain the same order of accuracy as in the tests with a lower number of TF-coils. Also, the current in the coil has to be proportionally com- 
Bootstrap current densities with changing detuning factor

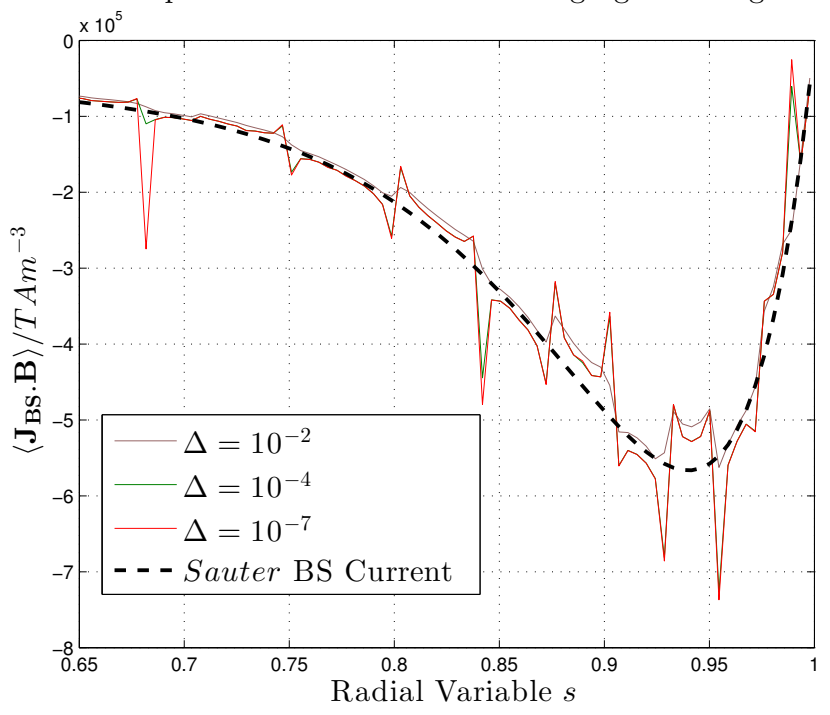

FIG. 7. The parallel Shaing-Callen bootstrap current density $\left\langle\mathbf{J}_{\mathrm{BS}}\right.$.B. $\rangle$ for different values of detuning factor $\Delta$. The dashed black line in the background is the Sauter bootstrap current calculated for $\Delta=10^{-7}$. The bootstrap current density curve for $\Delta=10^{-7}$ overlaps with the curve for $\Delta=10^{-4}$.

\section{Coil placement in MAST}

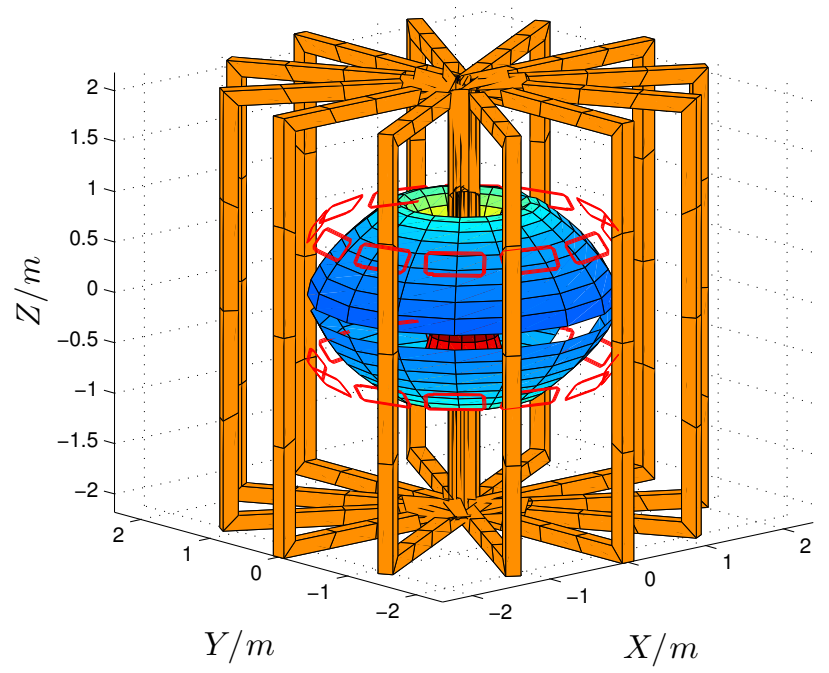

FIG. 8. Diagram depicting coil placement in MAST. The doughnut shaped figure is a plot of the magnetic field on the last closed flux-surface of the equilibrium. The toroidal field coils are shown in orange, and the RMP coils are shown in red.
Bootstrap current densities with number of TF-coils

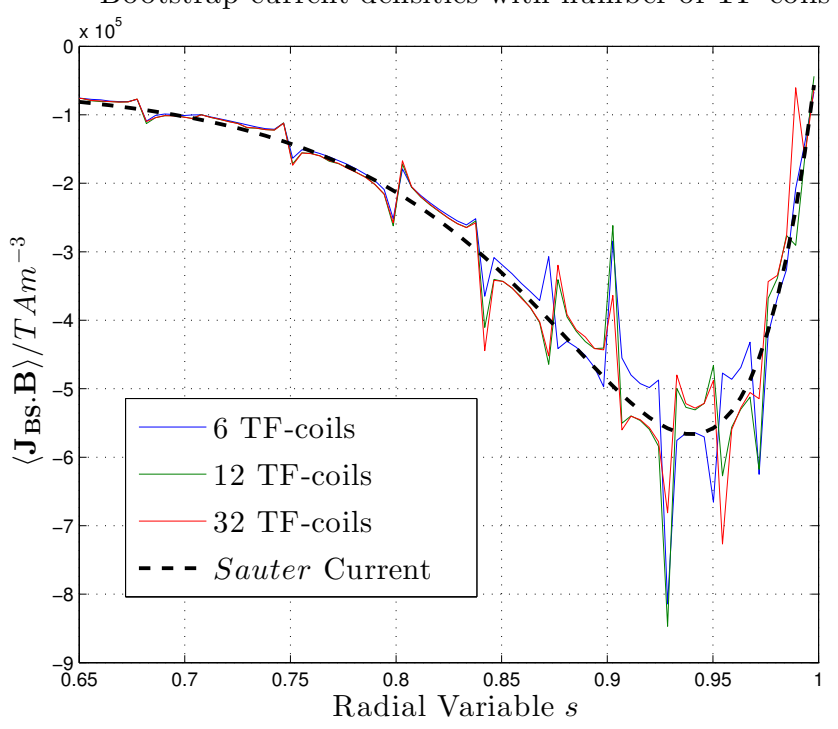

FIG. 9. The effect of increasing TF-coil numbers on the parallel Shaing-Callen bootstrap current densities. The resonance detuning factor is fixed at $\Delta=10^{-4}$. The black dashed curve represents the Sauter bootstrap current density for the same $\Delta$.

pensated corresponding to the number of coils being used in order to maintain the same field strength.

We notice from Fig. 9 that when the number of TFcoils is decreased to 6 , there is a change in the bootstrap current profile, though the net difference from the axisymmetric value does not significantly increase. In addition, we can see more spikes for the case with 6 TF-coils, which implies that a larger ripple causes more resonances, and hence more spikes. However, as can be seen in the figure, these additional spikes appear at mid-radius region, where the bootstrap current is weak. However, the increase in the number of TF-coils beyond 12 neither affects the bootstrap current density curve, nor the particular spikes observed in the density profile. Thus, we conclude that the number of TF-coils does not play any significant role on the form or order of the bootstrap current. In all the cases beyond 12 TF-coils, we do not see a difference in the bootstrap current density curve.

For all the cases considered, including the case with 6 TF-coils where a strong ripple ensues at the edge of the plasma, the Shaing-Callen bootstrap current density curve closely follows the axisymmetric Sauter bootstrap current density curve, except for the spikes observed at the resonant $q$-rational surfaces. It is reasonable to hypothesize that if these spikes were not present, the curves agree closely with the axisymmetric case. It is therefore reasonable to conclude that the axisymmetric Sauter model is a good representation of the bootstrap current, for the cases with a steep edge pedestal and toroidal field ripple. This is essentially because the plasma is nearly 


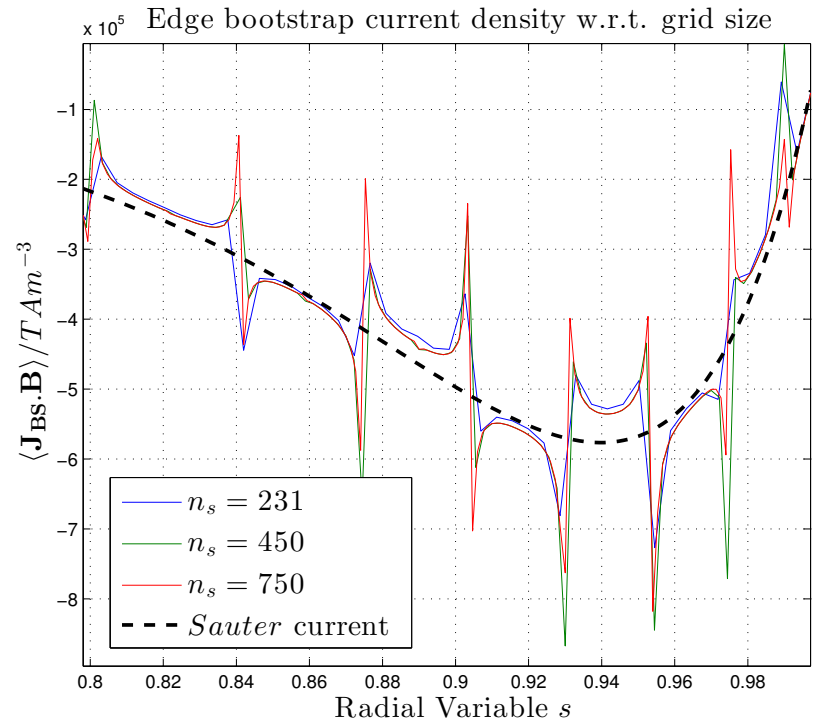

FIG. 10. A plot of the bootstrap current density, for the case of $32 \mathrm{TF}$ coils, for increasing sampling in radial variable $s$. We have especially zoomed in between the near-edge region $s=0.8-1.0$. It is immediately noticeable that the increase in the sampling produces neither a significant amelioration of the numerical resonance, nor do the non-resonant portions differ significantly.

axisymmetric.

Furthermore, we investigate whether an increased VMEC coordinate grid-size can help mitigate the resonant spikes. For this purpose, we fix the TF-coil number at 32 in order for VMEC to be able to resolve the magnetic field better near the edge, keep the RMP coil currents at $0 k A$, and proceed to increase the radial resolution $n_{s}$. We find, as is noticeable in Fig. (10), that the radial sampling also plays no significant role in mitigating the resonances. Given that the computation time for VMEC scales as $n_{s}^{2}$, and considering that the increased radial sampling does not affect the bootstrap current density curve, we conclude that lower grid-sizes provide sufficiently converged bootstrap current density values with the added benefit of a significantly lower computation time. (We essentially reproduce the grid-size effects in Ref. 9, but for presence of resonant current sheets in bootstrap current density calculations.)

Additionally, we would like to learn if any relevant physics can yet be extracted under similar conditions. Given that RMPs play an important role in mitigating ELMs, the effects of RMP fields on the bootstrap current are important. We apply RMPs to the same VMEC equilibrium (12 TF-coils) and get equilibria corresponding to the ideal response of the RMP fields. The RMP coils, again, are specified through the MAKEGRID package. Now, in order to check the effect of the RMP fields on the bootstrap current, we progressively increase the value of

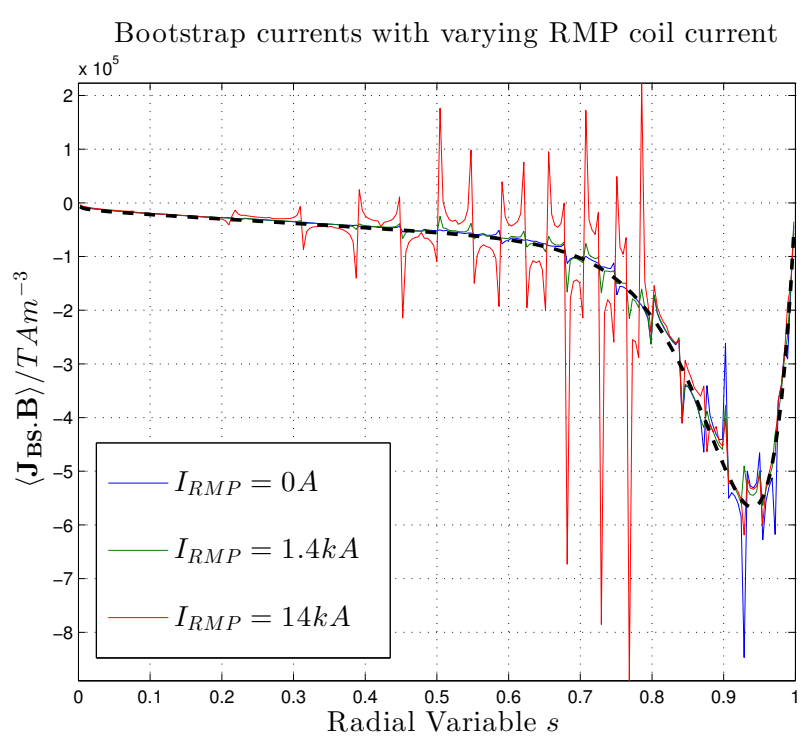

FIG. 11. The parallel Shaing-Callen bootstrap current density $\left\langle\mathbf{J}_{\mathbf{B S}} . \mathbf{B}\right\rangle$ as calculated for $12 \mathrm{TF}$-coils for a MAST 3D equilibrium with varying RMP currents. The black dashed curve represents the Sauter bootstrap current. The resonance detuning factor is set to $\Delta=10^{-4}$. Note in particular the higher $(m, n)$ mode number resonances caused in the midradius.

the bootstrap current from $0 k A$ to $14 k A$. In MAST, the order of currents used in the RMP coils is usually $1 k A$. In our scan, the value closest to realistic values would be around $1.4 k A$. It is worth mentioning that the $14 k A$ case is purely academic, pertaining to curiosity regarding any extreme effects RMPs might cause to the edge bootstrap current. The RMP coils, as seen in Fig. 8 are chosen so as to create an $n=3$ perturbation of even-parity respecting stellarator symmetry required of the up-down symmetric plasmas considered.

We notice that beyond a certain value of the RMP current, there is virtually no difference in the bootstrap current at the edge, and that the bootstrap current remains virtually unchanged between the RMP-coil current values of $I_{R M P}=0 A$ and $I_{R M P}=1.4 k A$. The RMP-coil current values lying in between these values correspond to a bootstrap current curve that lies in between the blue and green curves in Fig. 11 .

We notice that the bootstrap current at the edge is not significantly modified by varying the RMP current. However, in the $s=0.3$ to $s=0.8$ region, there is a small but noticeable effect of the changing $I_{R M P}$ on the 3D ShaingCallen bootstrap current. It is indeed expected that an $n=3$ perturbation would penetrate more towards the axis. Ultimately however, we conclude a negative result, that RMPs do not affect the bootstrap current significantly for realistic RMP coil currents. In contrast, if we assume (an unrealistic) value of RMP-coil current at $I_{R M P}=14 k A$, we see that the parallel current spikes in the region between the edge and the axis is more affected 
than the edge itself. We see, in general, that the parallel current spikes grow with the increasing RMP-coil current, therefore distorting the bootstrap current profile significantly and preventing us from extracting any significant results in this region of interest. Again, the 3D model does not offer any insight here. To summarize, we observe no significant change in the bootstrap current density with any value of RMPs in the edge region and the effects of the $n=3$ RMP on the bootstrap current density in the mid-radius region are masked by strong higher-order resonances. Thus the axisymmetric model is as good as the 3D model for the 3D equilibria with steep edge pressure barrier.

\section{BOOTSTRAP CURRENT PROFILES FOR A 3D HELICAL-CORE CASE WITH WEAK INTERNAL PRESSURE GRADIENT}

A saturated $n=1$ kink is known in experimental plasma physics as a long-lived mode (LLM) $\frac{10111}{1}$ or a helical-core. The deformation of the flux-surfaces near the core twist around helically with the toroidal angle. The helical core extends up to the point where the safety factor $q$ is at its minimum $\left(q_{\text {min }}\right)$, i. e. where the rotational transform is maximum $\left(\iota_{\max }\right)$. Beyond that, the flux-surfaces are almost axisymmetric, and the region enclosing the helical core is known as the axisymmetric mantle. LLMs play an important role in the functioning of machines such as MAST and JET, and future fusion devices like ITER. Helical-core equilibria can be generated in VMEC by initializing a VMEC simulation with an initial magnetic axis guess with a $1 / 1$ distortion which leads to the formation of a helical core.

We now begin by fixing the safety factor $q$-profile in VMEC, and letting the current profile relax in VMEC. In order to avoid resonance, we choose a $q$-profile which avoids major resonant surfaces in the helical-core region and which possesses a minimum $q_{\min } \approx 1$. Additionally, the pressure profile is chosen so as to provide a weak internal pressure barrier in the helical core region. Having fixed the $q$-profile, and not the current density profile $2 \pi\langle\mathbf{J} . \nabla \boldsymbol{\Phi}\rangle$, we only perform the first step of the iteration to obtain the form of the bootstrap current profile. Following from benchmarks in the preceding sections, the first iteration itself will bring the bootstrap current current density profile sufficiently close to the converged value. Additionally, the helical-core deformation can be observed more prominently in terms of the minor radius $r \sim \sqrt{s}$ rather than in the normalised toroidal flux $s$, henceforth, we plot all the relevant quantities in $\sqrt{s}$ in this section.

The $q$-profile is chosen to have a minimum around $s=0.2$ or equivalently, $\sqrt{s}=0.45$, indicating that the helical core extends until that point, as can be seen from Fig. 12. Beyond that, the equilibrium is effectively axisymmetric. Furthermore the pressure profile in the helical core-region is chosen to provide a weak pressure gradi-

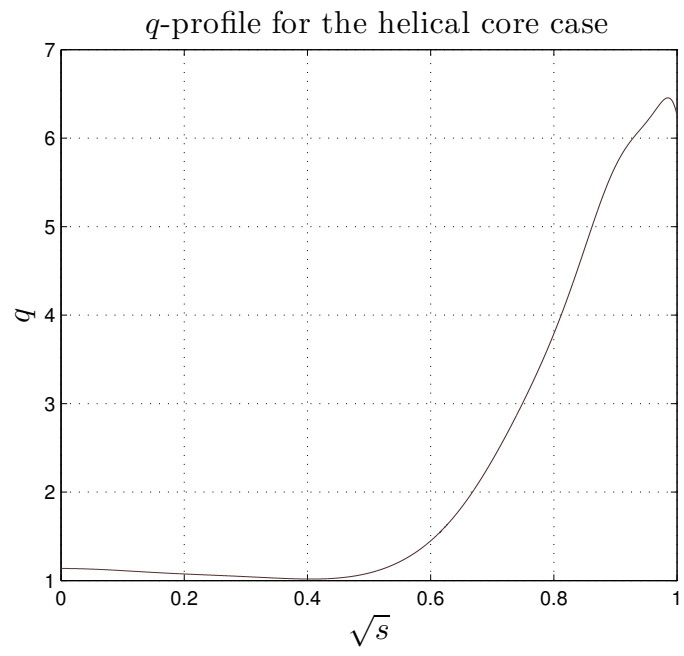

FIG. 12. The safety factor $q$-profile chosen to generate a helical core.

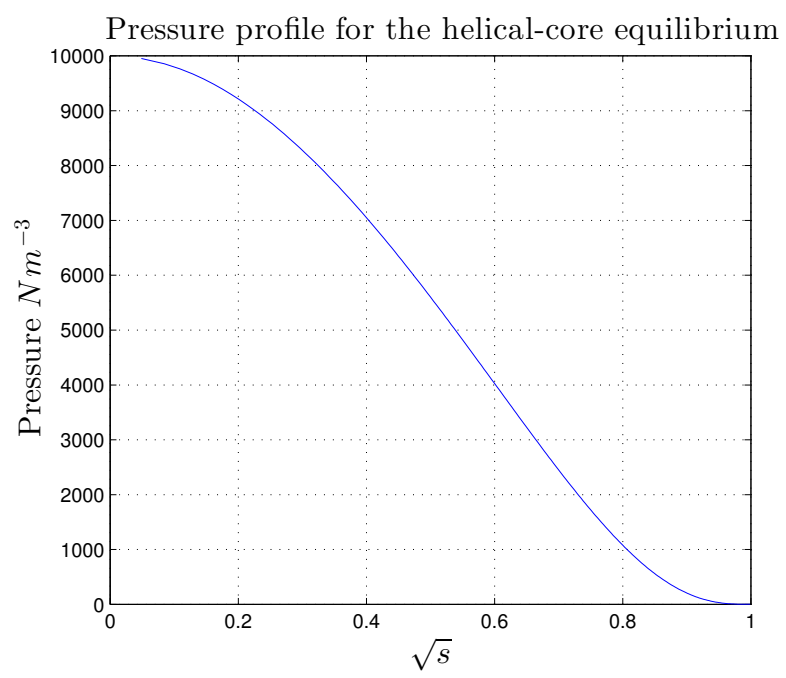

FIG. 13. The chosen density profile for the helical core equilibrium. We can see that in the helical core-region up to $s=0.2$, there is a pressure gradient, consistent with a weak internal pressure barrier.

ent in the helical-core region, as seen in Fig. 13 The temperature profile is chosen to be constant at $\bar{T}=640 \mathrm{keV}$, thus making the pressure profile of the same form as the density profile. With these, we generate a 3D equilibrium with a helical core, which can be seen in Fig. (14).

Using the above-mentioned profiles, we start by benchmarking the case against a 2D axisymmetric sister-state. In order to create this axisymmetric sister equilibrium, we force only one toroidal mode $n=0$, which forces VMEC to have an axisymmetric magnetic axis around which to form an equilibrium. This axisymmetric equilibrium is similar in all aspects with its helical core sister-state except for the presence of a helical skew in the core up to around $s=0.2$, which can be seen in Fig.15. We per- 


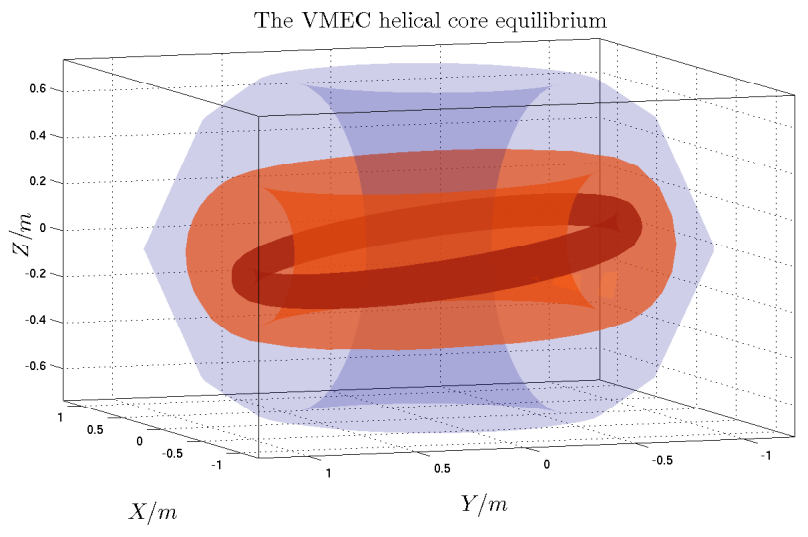

FIG. 14. The helical core equilibrium obtained from VMEC for the specified $\iota$-profile and pressure profile. One surface from inside the helical core $(s=0.1)$, one from near the axisymmetric boundary $(s=0.25)$ and the last from the edge $(s=1)$ are shown here for reference.

form our bootstrap current density calculations on this axisymmetric equilibrium using the Sauter and ShaingCallen equilibrium. One can see in the Fig. (16), that again, we see an excellent agreement between the two models to within 5\%. Clearly, the form of the Sauter bootstrap current density is determined chiefly from the pressure gradient $d p / d s$ and the trapped fraction $f_{t}$. In the core region $s<0.2$, the trapped fraction $f_{t}$ is the chief contributor to the form of the bootstrap current, taking it abruptly towards zero as it approaches the magnetic axis. Again, the Shaing-Callen bootstrap current density also closely follows the Sauter bootstrap current density. We observe no resonant contributions because of the lack of $n \neq 0$ modes in the computation of the geometrical factor $G_{b}$.

Having found a similar agreement as the previous edge pressure barrier case for axisymmetry, we move on to compute the bootstrap current density for the 3D helical core equilibrium with a skewed magnetic axis and a nearly axisymmetric free boundary. With the carefully chosen $q$-profile to avoid major resonances in the helical core region, we compute the Sauter and Shaing-Callen bootstrap current densities. The resonance detuning parameter is set at $\Delta=10^{-4}$, as per the optimal detuning parameter value obtained in the previous case. We notice from Fig. 17, that in the helical core region, there are no major resonance contributions, leading to a smooth bootstrap current density curve with the Shaing-Callen prescription. However, at a small distance outside of the helical core, near the beginning of the axisymmetric mantle, we notice spikes caused by the $\iota$-profile crossing major resonant surfaces. In the axisymmetric mantle, there is an agreement between the two formulations similar to what was seen for the edge pressure pedestal case. Cru-

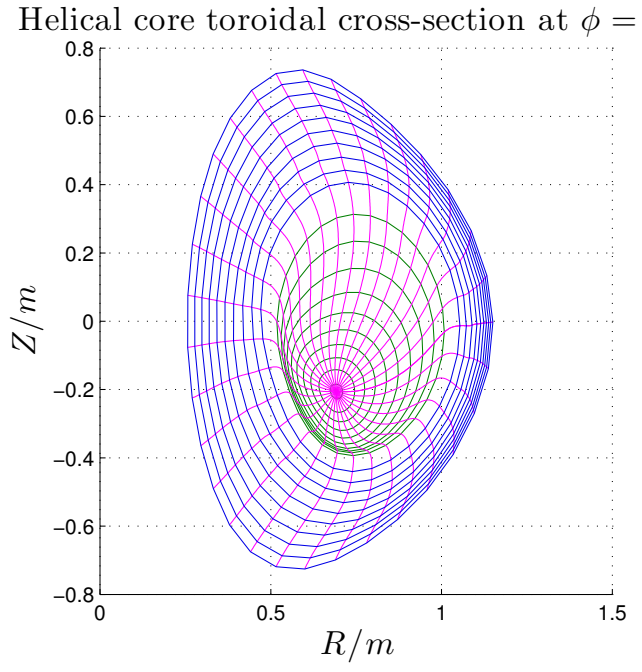

Axisymmetric analogue toroidal cross-section

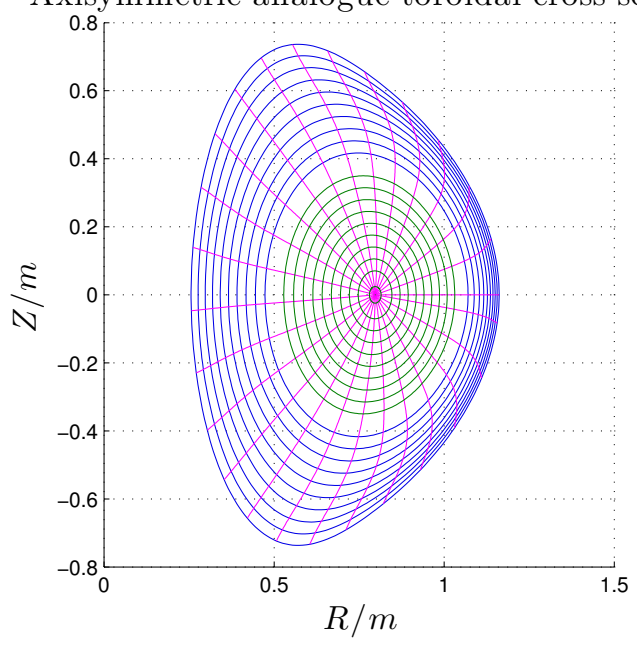

FIG. 15. A comparison of toroidal cross-sections of $(s, u, v)$ coordinate grids for the helical core equilibrium (above) and its axisymmetric sister-state (below). The magenta coloured lines are lines of constant $u$. The helical core region is represented by green coloured lines of constant $s$, and the axisymmetric mantle is represented by blue coloured lines of constant $s$.

cially, the bootstrap current densities prescribed by the Sauter formulation and the Shaing-Callen formulation in the helical-core region are visibly different. We proceed to investigate the origins of the difference between the two bootstrap current prescriptions in the helical core region.

The Sauter bootstrap current density depends on the values of $I(s)$, and the coefficients $L_{31}, L_{32}$ and $L_{34}$. From the Eqs. (1)-(4), it can be immediately noticed that the $L$-coefficients are dependent on the trapped fraction $f_{t}$. An approximation for the trapped fraction ${ }^{21}$ in terms of the triangularity $\delta$, the inverse aspect ratio $\epsilon$ is given 
Bootstrap current density for axisymmetric helical core analogue

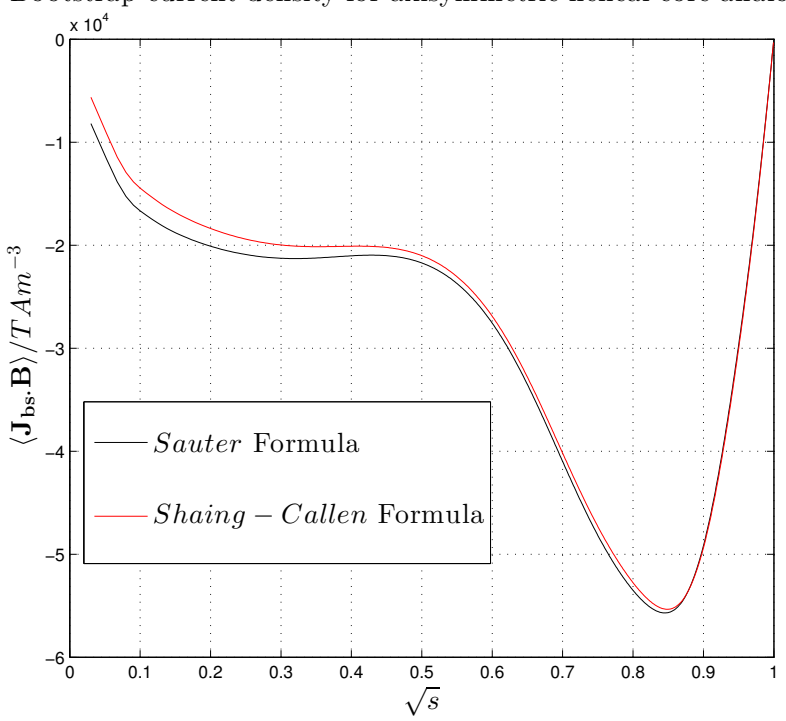

FIG. 16. The bootstrap current densities calculated for the axisymmetric sister state of the helical core equilibrium for the chosen helical core parameters.

by

$$
\begin{aligned}
\epsilon_{\text {eff }} & =0.67(1-1.4 \delta|\delta|) \epsilon, \\
f_{t, \text { approx }} & =1-\frac{1-\epsilon_{\text {eff }}}{1+2 \sqrt{\epsilon_{\text {eff }}}} \sqrt{\frac{1-\epsilon}{1+\epsilon}}, \\
f_{t, \text { approx }} & =\min \left(1, f_{t, \text { approx }}(\mathrm{Eq} \cdot 24) .\right.
\end{aligned}
$$

Now since $s=(r / a)^{2}, r$ and $a$ being the minor radius and maximum minor radius respectively, and thus $r / R$ being the aspect-ratio of the flux-surface in concern, we compute the approximate axisymmetric trapped fraction $f_{t, \text { approx }}$, through the values of $\epsilon$ and $\delta$ obtained from the VMEC equilibria. In addition, it is also possible to calculate the exact flux-surface averaged trapped fraction for the given axisymmetric VMEC equilibrium using Eq. 6. A comparison between the exact trapped fraction and the approximate trapped fraction can be seen in Fig. (18). The two axisymmetric trapped fractions follow each other quite closely as expected. Importantly, the exact trapped fraction $f_{t}$ for the $3 \mathrm{D}$ helical core (Fig. (18)), $f_{t}$ does not approach zero towards the magnetic axis, and it is for this reason that the Sauter model yields non-zero bootstrap current on the axis (see Fig. (16)). For the 3D VMEC helical core equilibrium, the skewed magnetic axis has a variation in the radial $R$ and azimuthal $Z$ directions. This causes a variation in the absolute magnetic field $|B|$, and therefore allows for the formation of a local magnetic well on the magnetic axis where the particles can get trapped. By following the same approach as Ref. [22, and taking into account the movement of the magnetic axis in $(R, Z)$, we arrive at the following approximation for the trapped fraction on the magnetic
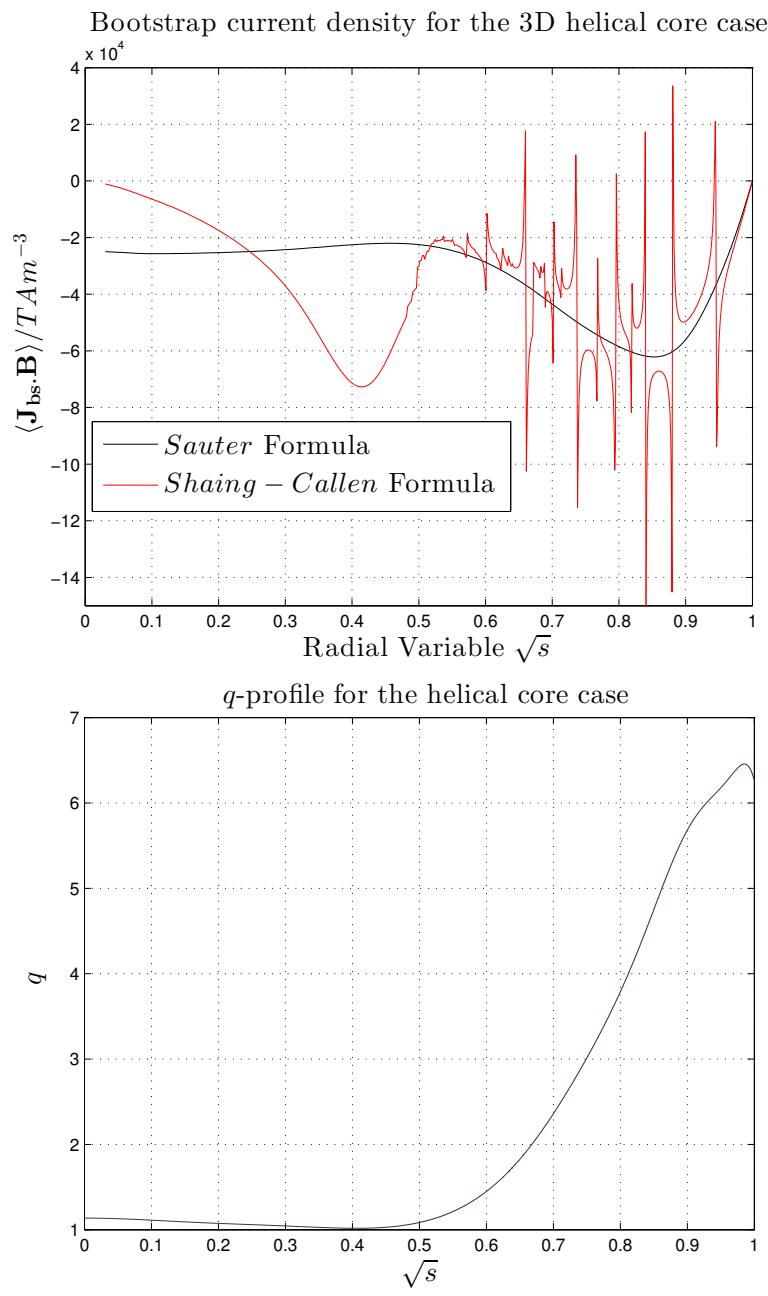

FIG. 17. The bootstrap current profiles obtained from the Sauter and Shaing-Callen formulations respectively (above), depicted for the $\iota$-profile (below).

axis

$$
f_{t, \text { approx }, \text { hel }}=\left(\frac{2 \Delta r_{h e l}}{R_{0}}\right)^{1 / 2}
$$

where $\Delta r_{h e l}$ is the the displacement of the magnetic axis. From the equilibrium data, we find $\Delta r_{h e l} \approx 0.18$, which makes the trapped fraction on the axis $f_{t, \text { approx }, \text { hel }} \approx$ 0.66, which is consistent with what we observe in Fig. 18 . The disparity in the 2D and 3D exact trapped fractions is very low in the axisymmetric mantle and therefore the agreement between the Sauter and Shaing-Callen formulations outside of the helical core region still remains of the order observed in Figures $(16)$ and $(17)$. It must also be mentioned that the collisionality towards the magnetic axis is typically not negligible, and the trapped fractions should realistically be reduced by the order of the collision frequency of the species ${ }^{3}$. This would help drive the trapped fraction to a lower value near the magnetic axis 


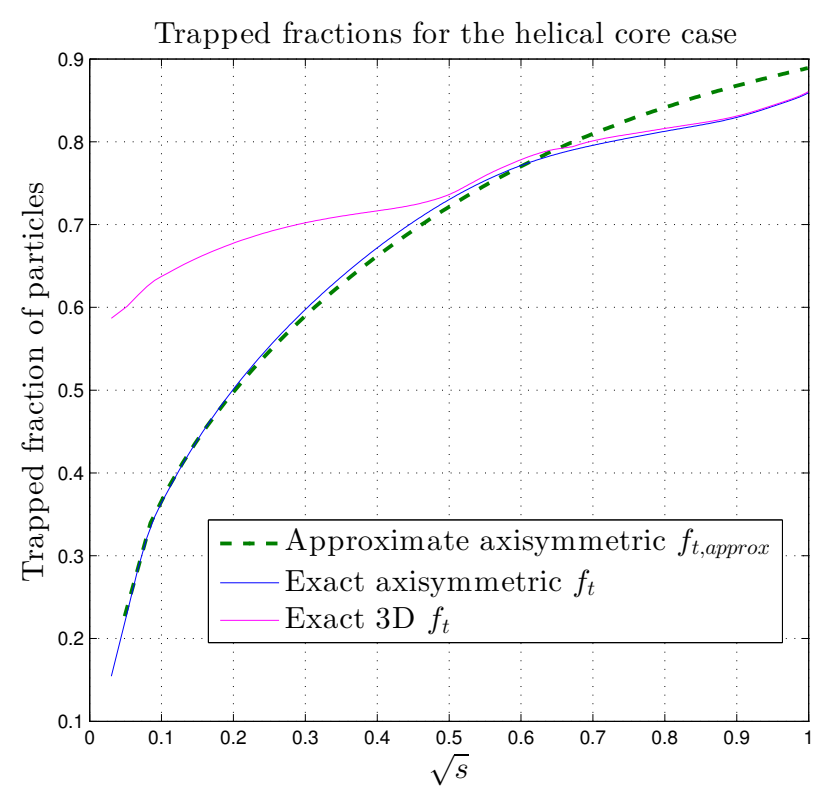

FIG. 18. A comparison between the exactly calculated trapped fraction $f_{t}$ for the $2 \mathrm{D}$ and $3 \mathrm{D}$ equilibrium and the trapped fraction $f_{t, \text { approx }}$ calculated from Eqs. (23)-(25).

for the 3D case, which would in turn modulate Sauter bootstrap current density to a lower value at the axis. However, this wouldn't affect the shape of the current density curve away from the magnetic axis, and the difference observed among the two bootstrap models will remain significant as the current case collisionless case considered.

As for the Shaing-Callen bootstrap current density formulation, the coefficients again depend on the neoclassical viscosity coefficients and the trapped fractions. However, as we have seen earlier, the trapped fractions do not approach zero, and from Ref.17, the viscosity coefficients are constants. Therefore, the key contribution to the modulation of the form of the bootstrap current density curve arises from the geometrical factor $G_{b}$, a factor that is not accommodated for in the Sauter model. From Fig. (19), the geometrical factor approaches zero towards the magnetic axis. The geometrical factor $G_{b}$, as evidenced from Eqs. (6) and (9)-(12), depends on the fluxsurface averaged coefficients $\left\langle g_{2}\right\rangle$ and $\left\langle g_{4}\right\rangle$, which subsequently depend on B. $\nabla \mathbf{B}$ and $\mathbf{B} . \nabla g_{1}$ respectively. For axisymmetry, the value of $|B|$ on the magnetic axis is constant along the poloidal angle $\theta$ and the toroidal angle $\phi$. Therefore, from Eqs. (12)-(13), $g_{2}, g_{4}$ and their flux-surface averages on the axis remain zero, leading the geometrical factor $G_{b}$ to a null value. In the $3 \mathrm{D}$ case, exploiting the symmetry in $\theta$, the RHS of Eq. (10) and Eq. (11) on the magnetic axis can be written as

$$
\mathbf{B} \times \nabla \Phi . \nabla \rightarrow \frac{g_{\psi \phi} B_{\theta}}{\sqrt{g}} \frac{\partial}{\partial \phi}
$$

where $\sqrt{g}$ is the Jacobian, $g_{\psi \phi}$ is the metric element
Geometrical Factor $G_{b}$ for helical core cases

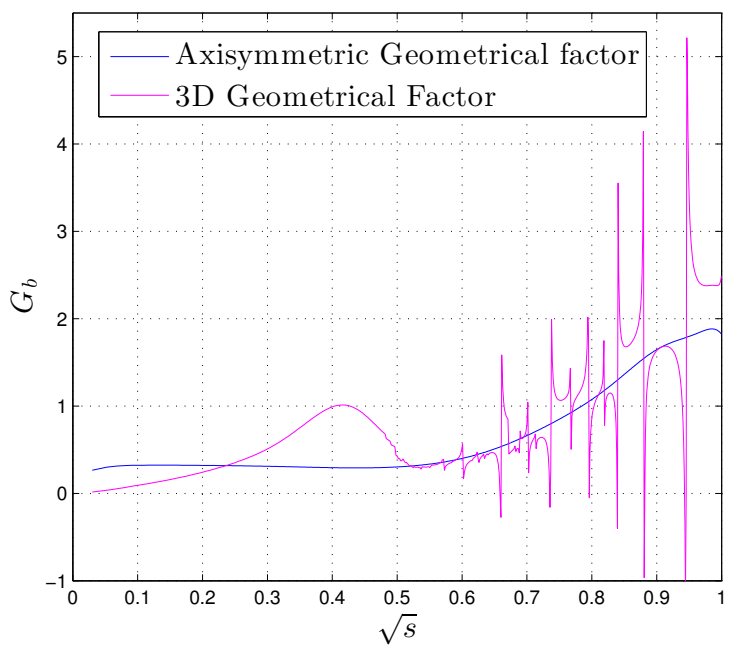

FIG. 19. The geometrical factor $G_{b}$ for the Shaing-Callen model. The geometrical factor goes to zero on the magnetic axis, and it exhibits the resonances observed in the axisymmetric mantle.

$\partial R / \partial \Phi$ and $B_{\theta}$ is the covariant $\theta$ component of the magnetic field. The Jacobian $\sqrt{g}$ is symmetric with $\phi$, and $B_{\theta}$ is constant with $\phi$. However the metric element $g_{s \phi}$ (and equivalently $\partial R / \partial \phi$ ) is anti-symmetric with $\phi$ for the $1 / 1$ internal kink mode magnetic axis. Therefore, the overall product of $g_{\psi \phi}, B_{\theta}$, and $1 / \sqrt{g}$ is anti-symmetric toroidally, leading to the integrals for $g_{2}$ and $g_{4}$ to be toroidally anti-symmetric. This leads to the flux-surface averages $\left\langle g_{2}\right\rangle=0$ and $\left\langle g_{4}\right\rangle=0$, thus ensuring that the geometrical factor $G_{b}$ goes to zero on the magnetic axis. Thus, the geometrical factor $G_{b}$ for the Shaing-Callen model will always approach zero at the magnetic axis for a 1/1 saturated internal kink mode, giving a significantly different result from the Sauter model.

The summary of this comparison can be seen in Fig. (20). It is evident that drastic drop in the Sauter bootstrap current density between $s=0$ and $s=0.1$ follows the trapped fraction $f_{t}$ profile. In contrast, the Shaing-Callen bootstrap current density follows the modulation offered by the geometrical factor. Thus the 3D model provides a physical resolution that the axisymmetric model falls short of.

We can thus conclude that, for a helical-core case which avoids resonant surfaces, it is important to choose the bootstrap model carefully. The difference between the Sauter and Shaing-Callen models, along with the possibility to choose non-resonant $\iota$-profiles, make the helical core a useful and important application in which the Sauter and Shaing-Callen models can show significantly different bootstrap current density profiles in the helical core region, a strong intrinsic 3D effect. 
Bootstrap current density for the helical core case

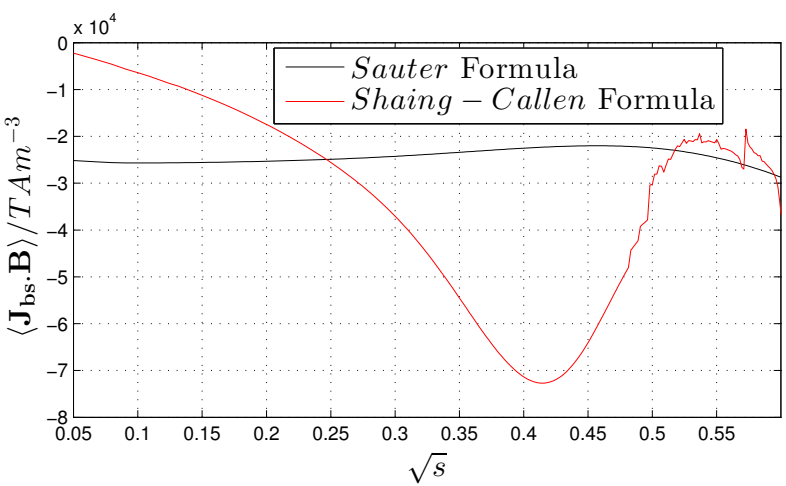

Trapped fractions for the helical core case

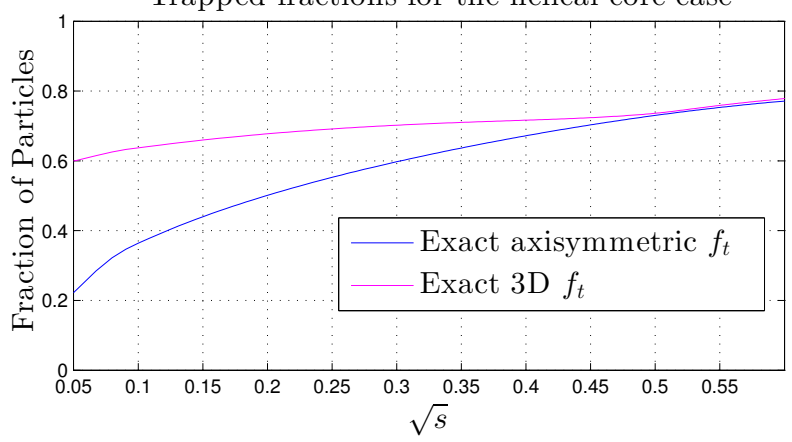

Geometrical Factors $G_{b}$ for helical core cases

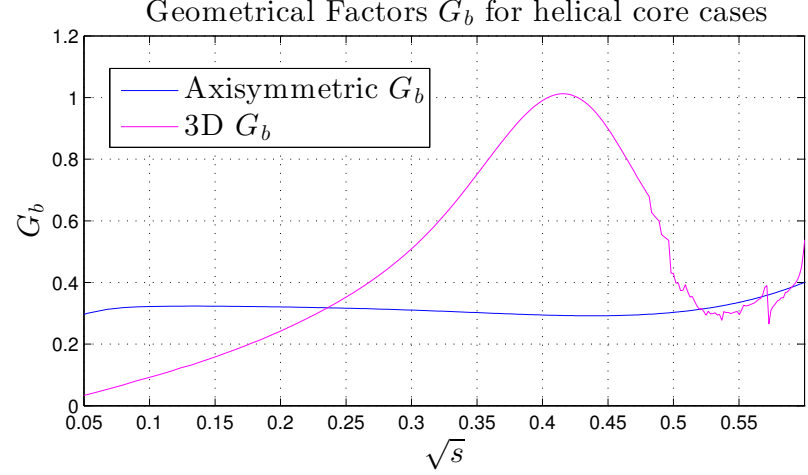

FIG. 20. A focus on the bootstrap current density in the helical core region. Also plotted are the trapped fractions and the geometrical factor for the Sauter and Shaing-Callen bootstrap current prescriptions against the causal factors.

\section{CONCLUSIONS AND THE OUTLOOK AHEAD}

We investigate the bootstrap current in MAST-like equilibria with an iterative self-consistent procedure in which the total current and the Ohmic current profiles are kept fixed. The bootstrap current is calculated from a given VMEC equilibrium and is adjusted into the toroidal current profile keeping the total current constant. This is iterated until convergence to specified tolerance. The bootstrap current profile is evaluated using using two known models: the Sauter and the Shaing-Callen models. We first began with a MAST equilibrium presenting a steep edge pressure pedestal as is observed in $\mathrm{H}$-modes.
For the axisymmetric test case considered, we observe good and rapid convergence. Being satisfied with the convergence and the self-consistency of the scheme in axisymmetry, we proceeded to examine $3 \mathrm{D}$ equilibrium with an edge pressure barrier with the two models. The 3D equilibrium presents severe current sheets at $q$-rational flux-surfaces, prompting an investigation into methods to minimize these resonances. First, when applying resonance detuning, we notice that the resonance detuning parameter $\Delta$ has a very narrow range of optimal values where the resonant $q$-rational surface currents are minimized without affecting the overall bootstrap current density curve. But the minimization of the parallel currents are still not enough to extract any useful physics from this case. In order to investigate ways to externally minimize the $q$-rational resonances, we proceeded to reduce the toroidal field ripple by increasing the number of TF-coils. We observe that toroidal ripple plays little effect when choosing more than 12 toroidal-field coils, implying that the axisymmetric model performs better than the 3D model in terms of avoiding the burden of parallel sheet currents caused on $q$-rational flux surfaces. In addition, we increase the sampling in the radial direction in order to minimize the width of the resonant current spikes. However, we observe that the increased sampling provides no significant improvement to the values of the resonances observed, leading to another negative result. In order to see whether some useful physical effects would still be salvageable from this particular 3D case, we apply RMPs, varying the current in the RMP coils. At the edge, where bootstrap current is the maximum for the edge pressure barrier based equilibrium, we observe virtually no change. This implies that the bootstrap current does not change its order of magnitude under RMPs. However, any significant effects caused by RMPs in the mid-radius region are again masked by severe current spikes. We conclude that the Shaing-Callen model is not useful for discerning any $3 \mathrm{D}$ effects on the bootstrap current density arising from toroidal field ripple or RMPs for the edge pedestal case.

In the remaining study, we choose to focus on choosing a $q$-profile that leads to a very strong $3 \mathrm{D}$ equilibrium in the core region. This application is relevant to Long Lived Modes in tokamaks $\frac{10}{10}$. We derive through VMEC, a finely tuned equilibrium with a helical core, by imposing a $q$-profile that avoids low order resonances in the helical core region. In the helical core region, we observe a significant difference between the Sauter and Shaing-Callen formulations. We conclude that these differences happen on account of the differences in the physical model, and that the 2D and 3D models do indeed present significantly distinguished results without resonance effects, making helical core studies an ideal candidate for bootstrap current research using the self-consistent iterative method. 


\section{ACKNOWLEDGMENTS}

This work has been carried out within the framework of the EUROfusion Consortium and has received funding from the Euratom research and training programme 2014-2018 under grant agreement No. 633053. The views and opinions expressed herein do not necessarily reflect those of the European Commission. The project was also supported in part by the Swiss National Science Foundation. The authors thank Dr. S. P. Hirshman for providing us with the VMEC equilibrium code. This work was supported by EPFL through the use of the computational facilities of the Scientific IT and Application Support Center (EPFL SCITAS).

${ }^{1}$ S. Coda, O. Sauter, M. A. Henderson and T. P. Goodman, 34th EPS Conference on Plasma Physics, EPS 2007 31, 2230 (2007).

${ }^{2}$ S. P. Hirshman, Physics of Fluids 26, 3553 (1983).

${ }^{3}$ O. Sauter, C. Angioni and Y. R. Lin-Liu, Physics of Plasmas 6, 2834 (1999) and 9, 5140 (2002).

${ }^{4}$ K. Shaing and J. Callen, Physics of Fluids 26, 3315 (1983).

${ }^{5}$ K. Y. Watanabe, N. Nakajima, M. Okamoto, K. Yamazaki, Y. Nakamura, and M. Wakatani, Nuclear Fusion 35, 335 (1995).

${ }^{6}$ M. Yu. Isaev, W. A. Cooper, K. Y. Watanabe, and N. Nakajima, Proceedings of the 30th Conference on Controlled Fusion and Plasma Physics 27, 4, (2003).

${ }^{7}$ K. Shaing, S. .P. Hirshman, and J. Callen, Physics of Fluids 29, 521 (1986).

${ }^{8}$ S. P. Hirshman, W. I. van Rij, and P. Merkel, Computer Physics Communications 43, 143 (1986).

${ }^{9}$ A. Wingen, N. M. Ferraro, M. W. Shafer, E. A. Unterberg,
J. M. Canik, T. E. Evans, D. L. Hillis, S. P. Hirshman, S. K. Seal, P. B. Snyder, and A. C. Sontag, Plasma Physics and Controlled Fusion 57, 104006 (2015)

${ }^{10}$ I. T. Chapman, M. D. Hua, S. D. Pinches, R. J. Akers, A. R. Field, J. P. Graves, R. J. Hastie, C. A. Michael, and The MAST Team, Nuclear Fusion 50, 045007 (2010).

${ }^{11}$ I. T. Chapman, D. Brunetti, P. Buratti, W. A. Cooper, J. P. Graves, J. R. Harrison, J. Holgatem S. Jardin, S. A. Sabbagh, K. Tritz, the MAST and NSTX Teams and EFDA-JET Contributors, Nuclear Fusion 54, 083007 (2014).

${ }^{12}$ W. A. Cooper, J. P. Graves, A. Pochelon, O. Sauter, and L. Villard, Physical Review Letters 105, 035003 (2010).

${ }^{13}$ D. Brunetti, J. P. Graves, W. A. Cooper and D. Terranova, Nuclear Fusion 54, 064017 (2014)

${ }^{14}$ D. Pfefferlé, W. A. Cooper, J.P. Graves, and C. Misev, Computer Physics Communications 185, 3127 (2014).

${ }^{15}$ W. A. Cooper, Plasma Physics and Controlled Fusion 34, 1011 (1992).

${ }^{16}$ K. Y. Watanabe, N. Nakajima, M. Okamoto, Y. Nakamura, and M. Wakatani, Nuclear Fusion 32, 1499 (1992).

${ }^{17}$ J. L. Johnson, K. Ichiguchi, Y. Nakamura, M. Okamoto, M. Wakatani, and N. Nakajima, Physics of Plasmas 6, 2513 (1999).

${ }^{18}$ A. D. Turnbull, W. A. Cooper, L. L. Lao, and L.-P. Ku, Nuclear Fusion 51, 123011 (2011).

${ }^{19}$ W. A. Cooper, S .F. I. Margalet, S. J. Allfrey, J. Kisslinger, H. F. G. Wobig, Y. Narushima, S. Okamura, C. Suzuki, K. Y. Watanabe, K. Yamazaki, and M. Yu. Isaev, Fusion Science and Technology 46, 365 (2004).

${ }^{20}$ D. V. Anderson, W. A. Cooper, R. Gruber, S. Merazzi, and U. Schwenn, International Journal of High Performance Computing Applications 1, 34 (1990).

${ }^{21}$ O. Sauter, submitted to Fusion Engineering and Design, 2016.

${ }^{22}$ J. Wesson, Tokamaks, 4th Ed., Sec. 3.11, 123-125 (2011). 